Letter to the editor

International Journal of Biological Sciences

ISSN 1449-2288 www.biolsci.org 2007 3(2):108-110

(c) Ivyspring International Publisher. All rights reserved

\title{
Application of Nanotechnology in Cancer Research: Review of Progress in the National Cancer Institute's Alliance for Nanotechnology
}

\author{
Beeta Ehdaie \\ Department of Biomedical Engineering, School of Engineering and Applied Sciences, University of Virginia, Charlottesville, \\ VA 22904, USA \\ Correspondence to: Beeta Ehdaie, Email: be7v@virginia.edu
}

Received: 2006.11.27; Accepted: 2006.12.25; Published: 2007.01.25

Cancer has become the leading cause of death among Americans today. In 2006, over 500,000 Americans will die due to cancer [11]. Cancer is a highly complex disease to understand because it entails multiple cellular physiological systems such as cell signaling and apoptosis [10]. Thus, the most common cancer treatments are limited to chemotherapy, radiation, and surgery. Limitations in cancer treatment are a result of current challenges seen in cancer therapies today, including lack of early disease detection, nonspecific systemic distribution, inadequate drug concentrations reaching the tumor, and inability to monitor therapeutic responses. Poor drug delivery and residence at the target site leads to significant complications, such as multi-drug resistance.

Nanotechnology has the potential to offer solutions to these current obstacles in cancer therapies, because of its unique size (1-100nm) and large surfaceto-volume ratios [13]. Nanotechnologies may have properties of self-assembly, stability, specificity, drug encapsulation and biocompatibility as a result of their material composition [9]. The NCI Alliance for Nanotechnology in Cancer has identified the potential of nanotechnology, and aims to accelerate the application of nanotechnologies in cancer research and clinical development. This program is a comprehensive, systemized initiative encompassing both public and private sectors [8]. The program comprises of eight Centers of Cancer Nanotechnology Excellence (CCNE), twelve platform projects, and four interdisciplinary training programs across the nation [http://nano.cancer.gov]. Thus far, research programs within the Alliance have made remarkable advancements in nanotechnology-cancer research. The objective of this article is to provide a brief review of these advances.

In the April 2006 PNAS publication "Nanoparticle-aptamer bioconjugates for cancer targeting", Langer and Farhrokzad from the MIT-Harvard CCNE tested the effect of aptamer-targeted nanoparticles for treatment of prostate cancer. This novel approach of using aptamers as targeted ligands, on drugencapsulated nanoparticles, proved to be highly effec- tive in targeting cancer cells and decreasing tumor size [6]. Aptamers are DNA or RNA oligonucleotides that fold in tertiary conformations, which then are able to bind to targeting antigens [4]. They are advantageous because aptamers are fairly small in size and, therefore, do not significantly impact the particle's overall size. Also, aptamers are non-immunogenic and fairly stable with long circulation times in the body. Aptamers have high affinity and specificity, which is an important requirement in targeting $[5,6]$. The use of these docetaxel-encapsulated nanoparticle-aptamer bioconjugates demonstrated a decrease in tumor size from approximately $300 \mathrm{~mm}^{3}$ to $120 \mathrm{~mm}^{3}$, as seen in Figure 1. In addition to reducing tumor size, these targeting methods had fewer side effects on healthy cells within the body in comparison to current methods of chemotherapy [6].

A number of the projects within the Alliance are investigating methods to overcome the challenges in maintaining drug circulation and avoiding multi-drug resistance. Due to chemical compositions and lack of targeting, many current therapeutic agents are removed from the body's circulation by the reticuloendothelial system (RES) or immune system [3]. In their June 2006 publication, Amiji and Langer addressed these challenges by developing poly(ethylene oxide)modified poly(beta-amino ester) (PEO-PbAE) nanoparticles. They studied the efficacy and toxicity levels of paclitaxel when it was administrated through PEO-PbAE nanoparticles in mice, bearing human ovarian adenocarcinoma xenograft. The PEO-PbAE polymer nanoparticles were significantly more efficient in drug delivery, reducing toxicity and decreasing tumor growth rates in comparison to the paclitaxel aqueous solution [3].

The interaction between the $\mathrm{PbAE}$ polymeric core with the PEO modified surface gives particles hydrophilic PEO components that decreases opsonization signal binding, and thus particles are less prone to phagocytes and macrophages [1-3,14,16]. Particles were $\mathrm{pH}$-sensitive, allowing drug release only at certain $\mathrm{pH}$ conditions, similar to $\mathrm{pH}$ levels unique in tumor cells. As a result the PEO-PbAE nanoparticles 
demonstrated the greatest drug efficacy. After four weeks the PEO-PbAE nanoparticle had reduced tumor volume to $22 \%$ of the original volume, where as the aqueous solution only had reduced tumor volume to $44 \%$ [3]. As these nanoparticles improve drug circulation, they also decrease chances of multi-drug resistance in long-term therapies.

In further research, Kommareddy and Amiji recently published their work on using poly(ethylene glycol) (PEG) surface modified thiolated gelatin nanoparticles to test drug and gene efficacy on breast cancer cells. Studies demonstrated prolonged circulation due to the hydrophilic PEG-modified surfaces. The PEG-modified thiolated gelatin nanoparticles had a high half-life of $37.8 \mathrm{~h}$ in the tumor mass. Also, drug release was administrated through thiolated gelatin nanoparticles that are highly sensitive to reducing environments, similar to those found in tumor cells [12]. Through nanotechnology, nanoparticles can be modified in a numerous ways to prolong circulation, enhance drug localization, increase drug efficacy, and potentially decrease chances of multi-drug resistance.

Nanotechnology is also overcoming challenges of early detection and imaging in current cancer therapies. In July 2006, an article by Sathe et al. demonstrated the use of nanotechnology in cancer detection [15]. Current detection methods are restricted in spectrum range, penetration depth, cell targeting, and signal/noise clarity [9]. The Nie team at the
Emory/Georgia Tech CCNE has been focusing on the development of quantum dots to improve detection methods. In the study mentioned above, they developed dual-functioning beads comprised of quantum dots and iron oxide nanocrystals embedded in silica beads. These particles were able to target specific cells, due to the iron oxide crystals, and have high imaging qualities, due to the quantum dot component. Currently, they are determining ideal combinations of iron oxides and quantum dots that would give the best imaging qualities [15]. Through nanotechnology, highly sensitive and specific imaging agents are being developed with biocompatible and multi-functional properties.

The role of the NCI Alliance is to monitor research programs to ensure they are product-oriented and milestone driven $[8,9]$. Within the five-year funding period, the Alliance aims to increase advancement rates by conducting annual progress reports, monitoring development and encouraging collaboration across the Alliance. This program is unique because it forms a strong network among the top researchers in the nation and encourages research advancement, so projects may reach clinical trial stages in the near future [8]. As a result of the organization and framework of the Alliance, there are high prospects in developing highly efficient cancer therapies through the application of nanotechnology.

Figure 1. A. Prostate Cancer cells were induced in mice using LNCaP prostate epithelial cells. Tumor development was allowed for 21 days in all conditions. Tumor masses were studied over a 109 day period. Saline was used as the control in this case. Results demonstrated that the docetaxel-nanoparticle-aptamers reduced tumor volume to $120 \mathrm{~mm}^{3}$ from $300 \mathrm{~mm}^{3}$. The none-targeted docetaxel nanoparticles also reduced tumor mass, however not as significantly as the aptamer-targeted nanoparticles. B. Difference in tumor mass is visibly seen with images of the mice and corresponding tumors at the end point. Black arrows point to the position of the implanted tumor. (Source: Farokhzad et al. [6]. Figure copyrighted National Academy of Sciences, U.S.A. Permission obtained.)

A.

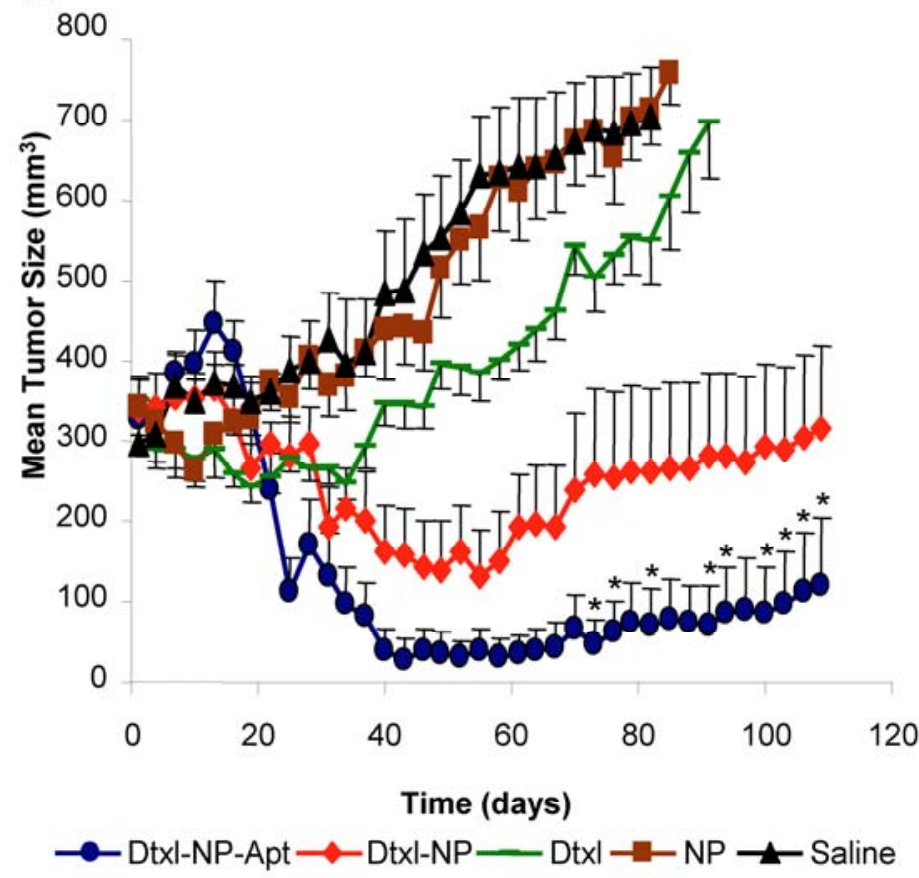

B.

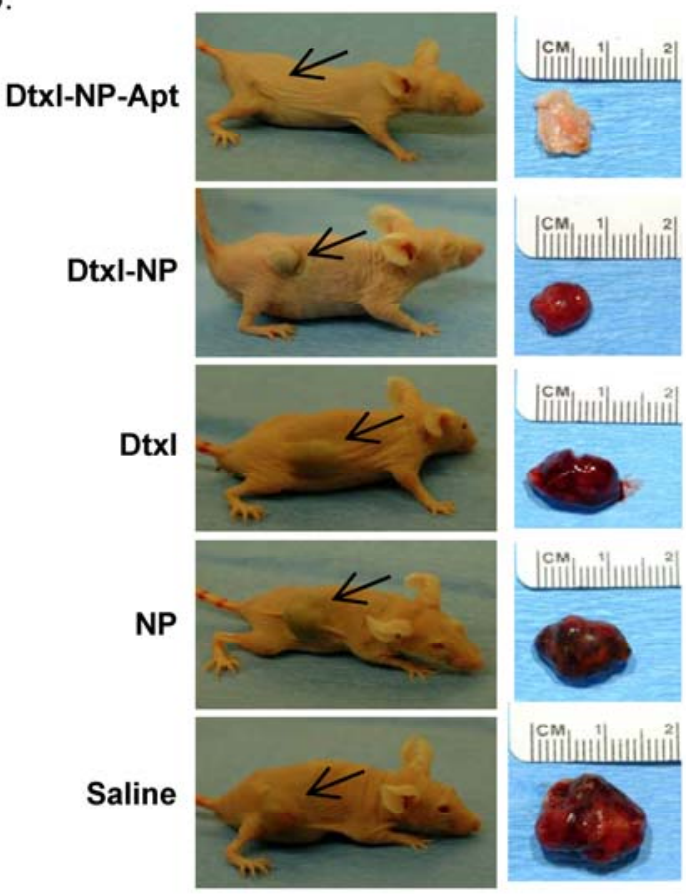




\section{Acknowledgements}

This review was supported by the 2006 National Institute of Health-Summer Internship Program, and conducted in the National Cancer Institute Alliance for Nanotechnology in Cancer, Office of Technology and Industrial Relations, Office of the Director, National Cancer Institute, 31 Center Drive, MSC 2580 Rm. 10A52. The author wishes to thank Dr. Chuxia Deng, Dr. Gregory Downing, Dr. Piotr Grodzinski, Dr. Jerry Lee, Mr. Travis Earles and Dr. Linda Molnar for their guidance and comments.

\section{Conflict of interests}

\section{Declared none.}

\section{References}

1. Amiji M, Park K. Prevention of protein adsorption and platelet adhesion on surfaces by PEO/PPO/PEO triblock copolymers. Biomaterials. 1992; 13: 682-692.

2. Amiji M, Park K. Surface modification of polymeric biomaterials with poly(ethylene oxide): a steric repulsion approach. In: Shalaby SW, Ikada Y, Langer R, Williams J (ed) Polymers of biological and biomedical significance. American Chemical Society symposium series publication. 1994; 540:135-146.

3. Devalapally H, Shenoy D, Little S, Langer R, Amiji M. Poly(ethylene oxide)-modified poly(beta-amino ester) nanoparticles as a $\mathrm{pH}$-sensitive system for tumor-targeted delivery of hydrophobic drugs: part 3 Therapeutic efficacy and safety studies in ovarian cancer xenograft model. Cancer Chemotherapy and Pharmacology; in press.

4. Ellington AD, Szostak JW. In vitro selection of RNA molecules that bind specific ligands. Nature. 1990; 346: 818-822.

5. Farokhzad OC, Karp JM, Langer R. Nanoparticle-aptamer bioconjugates for cancer targeting. Expert Opin. Drug Deliv. 2006; 3: 311-324.

6. Farokhzad OC, Cheng J, Teply BA, et al. Nanoparticle-aptamer bioconjugates result in significant tumor reduction in vivo. Proc. Natl Acad Sci USA. 2006; 103 (6): 6315-6320.

7. Ferrari M. Cancer Nanotechnology: Opportunities and Challenges. Nature Rev. Cancer. 2005; 5:161-171.

8. Ferrari M, Barker AD, Downing GJ. A Cancer Nanotechnology Strategy. NanoBiotechnology. 2005; 1:129-131.

9. Grodzinski P, Silver M, Molnar LK. Nanotechnology for Cancer Diagnostics: Promises and Challenges. Expert Rev. Mol. Diagn. 2006; 6 (3):307-318.

10. Hanahan D, Weingberg RA. The Hallmarks of Cancer. Cell.2000; 100: 57-70.

11. Jemal A, et al. Cancer Statistics 2006. CA Cancer J Clin. 2006; 56(2): 106-130.

12. Kommareddy S, Amiji M. Biodistribution and Pharmacokinetic Analysis of Long-Circulation Thiolated Gelatin Nanoparticles Following Systemic Administration in Breast Cancer-Bearing Mice. J Pharm Sci; in press.

13. McNeil, SE. Nanotechnology for the Biologist. Journal of Leukocyte Biology. 2005; 78: 585-594.

14. Moghimi SM, Muir IS, Illum L, Davis SS, Kolb-Bachofen V. Coating particles with a block co-polymer (polox-amine-908) suppresses opsonization but permits the activity of dysopsonins in the serum. Biochem Biophys Acta.1993; 1179: 157-165.

15. Sathe TR, Agrawal A, Nie S. Mesoporous Silica Beads Embedded with Semiconductor Quantum Dots and Iron Oxide Nanocrystals: Dual-Function Microcarriers for Optical Encoding and Magnetic Separation. Anal. Chemistry. 2006; 78: 56275632.

16. Tan JS, Butterfield DE, Voycheck CL, Caldwell KD, Li JT. Surface modification of nanoparticles by PEO/PPO block co- polymers to minimize interactions with blood components and prolong blood circulation in rats. Biomaterials.1993; 14: 823-833. 